\title{
A mathematical framework to describe the effect of beam incidence angle on metrics derived from airborne LiDAR: The case of forest canopies approaching turbid medium behaviour
}

\author{
Jean-Romain Roussel, Martin Beland, John Caspersen, Alexis \\ Achim
}

Version Post-print/Accepted Manuscript

\begin{abstract}
Citation Jean-Romain Roussel, Martin Beland, John Caspersen, Alexis Achim (published version) (2018). A mathematical framework to describe the effect of beam incidence angle on metrics derived from airborne LiDAR: The case of forest canopies approaching turbid medium behaviour. Remote Sensing of Environment. 209. 10.1016/j.rse.2017.12.006.

DOI https://doi.org/10.1016/j.rse.2017.12.006

Copyright/License

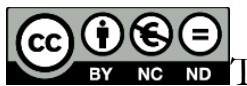

Cr ${ }_{\mathrm{NC}}$ ND This work is licensed under the Creative Commons Attribution-NonCommercial-NoDerivatives 4.0 International License. To view a copy of this license, visit Creative Commons BY NC ND 4.0 License.
\end{abstract}

\section{How to cite TSpace items}

Always cite the published version, so the author(s) will receive recognition through services that track citation counts, e.g. Scopus. If you need to cite the page number of the author manuscript from TSpace because you cannot access the published version, then cite the TSpace version in addition to the published version using the permanent URI (handle) found on the record page.

This article was made openly accessible by $U$ of $T$ Faculty. Please tell us how this access benefits you. Your story matters. 
Airborne laser scanning (LiDAR) is used in forest inventories to quantify stand structure with three dimensional point clouds. However, the 3D distribution of the point clouds depends not only on stand structure, but also on scan angle, because the probability for an oblique beam to be reflected by the canopy increases with the distance it must travel through the canopy. Thus, the canopy appears to increase in density as the incidence angle increases, all else being equal. The resulting variation between and within datasets can induce bias in LiDAR metrics derived from the vertical distribution of points. In this study, we modelled the effect of scan angle on the vertical structure of the point clouds to predict the bias of metrics derived from points sampled off-nadir. Comparison with paired observations from different flightlines (off- and at-nadir observations of the same point) demonstrated that the model accurately reproduced the bias of metrics calculated for a northern hardwood forest with relatively continuous canopy. Thus, the model could be used to correct the bias of LiDAR metrics, and provides a mathematical framework that could be used to inform the selection of maximum incidence angle in LiDAR surveys, considering the trade-off between decreasing acquisition costs and obtaining unbiased measurements. 
interpretation. Data processing relies partly on automated algorithms (e.g. Pyysalo and Hyyppä, 2002; Morsdorf et al., 2004; Reitberger et al., 2009; Kwak et al., 2010; Yao et al., 2012; Vega et al., 2014) and partly on empirical statistical models (e.g. Holmgren, 2004; Ioki et al., 2009; Lim et al., 2014; Bouvier et al., 2015). Height and density metrics derived from the point cloud can be used to estimate the horizontal and vertical distribution of vegetation, which have various applications in forestry and ecology (Vauhkonen et al., 2014). For example, forest managers use LiDAR to predict product recovery under different harvest prescriptions (Maltamo et al., 2014).

LiDAR has brought a fundamental improvement in the quality of aerial inventories, which explains the rapid uptake of this technology by practitioners (Popescu et al., 2002; Gleason and Im, 2012). It can be argued, however, that some aspects of this technology are not fully understood. For example, the literature does not provide adequate understanding of the effect of LiDAR sensor parametrization and flight pattern on the three-dimensional structure of the point cloud (Goodwin et al., 2007). Thus, empirical statistical models of stand structure may only be applicable to a single forest, a single device and a single set of acquisition parameters.

While locally calibrated models meet user needs at a given point in time, each new LiDAR survey may require another calibration with new ground data, given that both the device and acquisition parameters change through time. Thus, the use of LiDAR technology for forest monitoring requires a better understanding of how changes in device settings and flight patterns affect the structure of the point cloud. Ultimately, users would benefit from being able to normalize any two sets of lidar-derived metrics as if they were acquired the same way.

While several studies have been dedicated to understanding how the density of emitted pulses affects various metrics and their prediction accuracy (Lovell et al., 2005; Anderson et al., 2006; Thomas et al., 2006; Gobakken and Næsset, 2008; Lim et al., 2008; Pirotti and Tarolli, 2010; Jakubowski et al., 2013), few have examined the effect of incidence angle. Widening the scanning angle allows a larger area to be surveyed more rapidly and at a lower cost (Goodwin et al., 2007; Evans et al., 2009). However, the financial advantage of a wide scanning angle could be offset by significant biases in the derived metrics.

Despite uncertainties regarding its magnitude, the effect of scanning angle is unarguable when one considers the extreme case of a $89^{\circ}$ angle of incidence, in which case metrics are obviously biased compared to those obtained from a vertical beam simply because of shadowing effects or because of the increased distance between the top of the canopy and the ground. There is therefore a gradual increase in bias between 0 and $89^{\circ}$, such that 
the canopy appears to increase in density as the incidence angle increases, all else being equal. Yet, the physical or geometrical phenomena leading to such effects, the magnitude of these effects at a given angle, and their consequences are still largely unknown. Thus, the ongoing debate about the choice of the maximum incidence angle would benefit from a better mechanistic understanding of angular bias, so that the trade-off between cost and prediction bias could be quantified.

Holmgren (2004) recommended limiting the scanning angle to $10^{\circ}$ to prevent effects on forest metrics estimates, while Disney et al. (2010) proposed limiting it to less than $15^{\circ}$ to avoid ground detection problems. However, these suggestions are hardly applicable in the case of large survey areas and in practice the maximum incidence angle is typically $\pm 15-20^{\circ}$, with a will to increase it further.

No consensus can be drawn from studies that have attempted to quantify the effects of incidence angle on LiDAR-derived forest metrics. Holmgren et al. (2003b) and Lovell et al. (2005) provided simulations of non-divergent beams hitting conical, ellipsoidal or half-ellipsoidal solid (i.e. impermeable) digitally reconstructed trees. With such simplifications of the reality Lovell et al. (2005) showed a dependency of the predominant height of the canopy on maximum incidence angle. They pointed out that increasing the maximum incidence angle and keeping all other parameters unchanged leads to an overall decrease in pulse density. The measure of the predominant height was therefore biased, as was mathematically demonstrated by Roussel et al. (2017). Within a given scan, Holmgren et al. (2003b) showed that the percentiles of height tended to decrease with increasing incidence angle, using a simulation which assumed that trees were solid, impermeable objects. Lovell et al. (2005) also highlighted the importance of the incidence angle by showing that maximum tree height retrieval is less accurate at the scanning edges due to a more uneven spacing of LiDAR points. Goodwin et al. (2007) improved these simulations by using permeable half-ellipsoidal digital trees. Their results showed that larger incidence angles " produced a higher number of foliage hits and increased beam interception probability at the forest stand scale". They also demonstrated that higher incidence angles increase the crown area visible to a LiDAR pulse.

In their comparison of field measurements and LiDAR data, Holmgren et al. (2003a) did not find a statistically significant effect of the incidence angle on the estimation of a metric related to the height of dominant trees (i.e. mean of tree heights weighted by their basal area). Similarly, Næsset (1997) showed that the effect of the incidence angle was non-significant in a regression model used to predict forest basal area. These results do not necessarily contradict the previous results because all metrics may not be equally dependent on incidence angle. 
It is also possible that different effects can compensate for one another, so that some predictive models appear to be insensitive to the incidence angle. Morsdorf et al. (2008) also concluded that the effect of the incidence angle is not as evident as it may first appear.

Montaghi (2013) presented an interesting study on the effect of incidence angle, taking advantage of perpendicular flightlines -typically used for strip adjustment and calibration- to compare a large amount of data sampled at nadir and off nadir. Although the overly large number of t-test comparisons $(\sim 1300)$ implies that some of the statistically significant effects reported may only be attributed to random variation (1 out of 20 test at alpha $=0.05)$. Even with stronger statistical methods, this approach would lead to as many statistical models as metrics, i.e. one for each metric as they each have their own dependency to the incidence angle. Furthermore, these statistical models would be specific to a given dataset, which entails the same data-dependency issue described above.

An important issue with the current understanding of angular bias is that it has been acquired mainly through descriptive approaches. Indeed, mathematical models have rarely been used to explain how and why LiDAR metrics vary with incidence angle. Goodwin et al. (2007) and Disney et al. (2010) proposed that the probability a beam is reflected by the canopy increases as incidence angle increases, simply because the distance it travels through the canopy increases. Despite the plausibility of this explanation, a formal demonstration is still required.

In this study, we hypothesize that angular bias can be normalized by the distance a beam must travel through the canopy. We suggest a simple physical formalization of this hypothesis to quantify the effect of incidence angle on the vertical distribution of points. We then compare our theoretical model against real data to validate its relevance. Thus, rather than a very large number of empirical models derived for each metric, we propose a single overarching model that applies to all metrics.

\section{Material and methods}

\subsection{Study area}

The study area is located within the Haliburton Forest and Wildlife Reserve. The forest is a 32000 ha privately owned property located in the Great Lakes - St. Lawrence Forest Region of central Ontario, Canada (45ำ $13^{\prime}$ N, $78^{\circ} 35^{\prime}$ W). Elevation ranges from approximately 400 to $500 \mathrm{~m}$ above sea level. The forest is a mixture of hardwoods and 


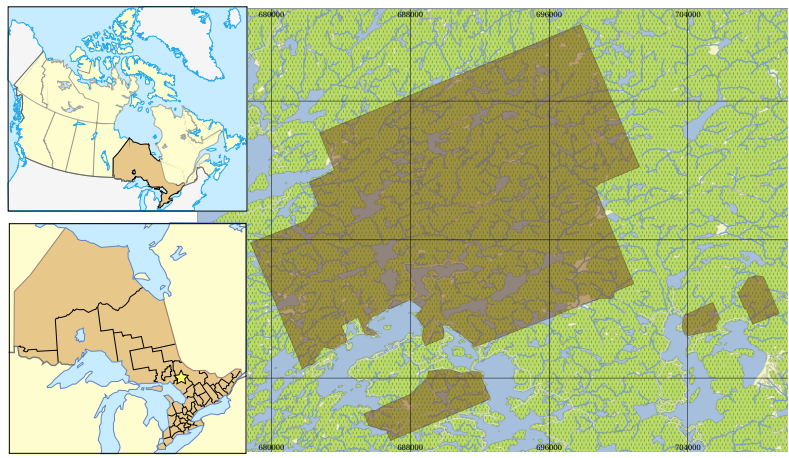

Figure 1: Map of study areas. Brown area represent the boudaries of the LiDAR dataset.

conifers typical of northern hardwood forests, and sugar maple (Acer saccharum Marsh) is the dominant species, comprising $60 \%$ of the basal area. Most of the forest has been managed under selection silviculture for the past 50 years, and was selectively harvested before then. Thus, most of the stands are uneven-aged, with average canopy heights ranging from 20 to $25 \mathrm{~m}$.

\subsection{LiDAR data}

The LiDAR dataset was acquired in August 2009 covering the whole $320 \mathrm{~km}^{2}$ area of the Haliburton forest. It was acquired with a pulse density of approximatively 2 pulses $/ \mathrm{m}^{2}$ on average. The complete set of parameters is given in table 1.

\subsubsection{Data pre-processing}

The normalization of the dataset (i.e. the subtraction of the digital terrain model) was done by the provider. The method was based on triangular irregular network construction from returns classified as "ground". Each point was interpolated, which implies that the normalization was not based on a digital terrain model, thereby giving a virtually infinite resolution. Further details about the algorithm used to determine point classes could not be obtained. We did not have access to the raw data.

Lakes and wetlands were filtered from the dataset in an attempt to retain only forested areas. The process was based on geographic data from the latest official cartography of Ontario, which spatially matched very closely with observed lakes and wetlands from our LiDAR datasets. 
Table 1: Flight parameters for the datasets. PRF: pulse repetition frequency

\begin{tabular}{lc}
\hline Parameter & Value \\
\hline \hline Sensor & Optech ALTM 3100 \\
Altitude & $1500 \mathrm{~m}$ \\
Swath overlap & $30 \%$ \\
Speed & $120 \mathrm{kts}$ \\
Scan Frequency & $36 \mathrm{~Hz}$ \\
System PRF & $70 \mathrm{kHz}$ \\
Max. off-nadir angle & $16^{\circ}$ \\
Cross track resolution & $0.89 \mathrm{~m}$ \\
Along track resolution & $0.86 \mathrm{~m}$ \\
Point density & $\approx 2 \mathrm{~m}^{-2}$ \\
Pulse density & $\approx 1.6 \mathrm{~m}^{-2}$ \\
Footprint size & $0.14 \mathrm{~m}^{2}$ \\
\hline
\end{tabular}

\subsection{Conceptual framework}

We aim to quantify the changes in the height distribution of returns that result from increasing the incidence angle, and hence both the distance a beam travels through the canopy and the probability that the beam is reflected by the canopy. In particular, we aim to develop a model that reproduces the resulting increase in canopy returns and the corresponding decrease in ground returns. Successful description of this incidence angle effect should enable normalizing the point distribution, and consequently every existing metric derived from elevations within the point cloud (i.e. classical metrics derived from $z$ coordinates) as if all data had been sampled at-nadir.

Our approach consisted of developing a set of two mathematical expressions to predict how a point distribution sampled at-nadir would be altered if it had been obtained from another off-nadir angle. To validate the model, the predicted bias of various LiDAR metrics derived from such an "off-nadir" point cloud was compared to the bias observed between paired observations from different flightlines. Among the infinite number of metrics that could be derived, we chose nine representative metrics that describe various aspects of a distribution. We believe that if our model is capable of predicting the behaviour of these diverse metrics, then it can be considered an adequate description of physical reality. 


\subsection{Metric computation}

We rasterized the dataset at the level of a "plot raster" i.e. a $20 \times 20 \mathrm{~m}$ pixel, which is a commonly used resolution both in the literature and in applications that map quantities of interest using an area based approach (Woods et al., 2011; White et al., 2013). The flightlines were treated individually to avoid introducing variation related to the existence of overlaps in which rasters were sampled twice. For each plot raster we computed one control metric (the mean absolute incidence angle of the returns) and, using all returns, nine metrics derived from the distribution of elevations in the point cloud:

- The mean height of the returns;

- The standard deviation of the heights;

- The coefficient of variation of the heights;

- The 30, 50 and $70^{\text {th }}$ percentiles;

- The kurtosis and the skewness of the distribution;

- The entropy of the height distribution as labelled in the context of information theory. This index often called the "Shannon index" or "Shannon evenness index" in forestry or ecology applications. van Ewijk et al. (2011) name it "vertical complexity index" in a paper dedicated to LiDAR.

These metrics were not chosen for their relevance in forest inventory applications, but instead because they represent classical descriptors of central tendencies, deviation and heterogeneity applicable to any distribution.

The mean absolute incidence angle was based on the scan angle rank i.e. the actual data stored according to LAS format specifications ASPRS (2013), which does not represent the real nadir angle. Zero may in reality be off-nadir, depending on aircraft roll angle. However, we did not consider this effect as it would only create negligible noise in our analysis.

\subsection{Software}

Data pre-processing and processing was done in the R programming environment (R Core Team, 2015). A package named lidR specifically developed for LiDAR data processing was used (Roussel and Auty, 2017). The R source code to compute the model is given as a supplementary material. 


\section{Model development}

\subsection{Initial considerations}

The model relies on probability theory and on three simplifying assumptions: the first two relate to the proportion of energy that is backscattered toward a LiDAR sensor, the third relates to the distribution and orientation of material within the canopy.

A quantity $E_{0}$ of energy emitted by a LiDAR instrument will either i) be absorbed by the canopy, ii) be backscattered in any direction other than towards the sensor, iii) be backscattered towards the sensor with insufficient energy to generate a point (when using discrete LiDAR) and, finally, vi) be backscattered towards the sensor with sufficient energy to generate a point. The first three quantities are considered to be "non-contributing", or "lost" energy, while the last quantity will be referred to as "contributing energy".

First, we assume that beams have an infinitesimal width and carry only contributing energy. Indeed, we worked with a point cloud that, by definition, resulted exclusively from such contributing energy. Following this assumption, when such a beam encounters an object of the canopy, it can only be reflected towards the sensor and generate a point. Multi-returns were considered to come from multiple beams and we neglected multiple scattering.

Second, we assume that the proportion of contributing energy is not affected by the beam incidence angle. Under this assumption, what changes between two different angles is not the amount of contributing energy but only the distribution of this energy throughout the canopy, and therefore the distribution of the triggered returns, or points.

Third, we assume that at a given elevation, the probability of reflecting contributing energy is proportional to the number of canopy elements, or material density. As we did not have prior information about the vertical distribution of canopy elements within a canopy layer, we assumed a random distribution at any given elevation.

Under these assumptions, the model describes how to recompute a point distribution sampled at one angle "as if it were sampled at another angle". It relies on a set of two equations - the gap fraction profile function as presented in Bouvier et al. (2015) and its reciprocal function.

\subsection{Notation}

$\theta$ incidence angle 
$I$ event "beam interacts with a canopy element"

$R$ event "beam is reflected"

$A$ event "beam is absorbed"

$X$ a random variable

$\bar{E}$ complementary event of the event $E$

$\mathscr{P}(E)$ probability of the event $E$

$p_{k}$ probability for a given beam to generate a point in layer $k$

$i_{k}$ probability of $I$ in layer $k$ for a given beam

$r_{k}$ probability of $R$ in layer $k$ for a given beam

$a_{k}$ probability of $A$ in layer $k$ for a given beam

$\Delta z$ layer thickness

\subsubsection{Gap fraction profile}

The gap fraction describes the probability for a beam to reach the ground without encountering canopy elements. Bouvier et al. (2015) proposed an equation adapted to point clouds for computing the gap fraction profile as a function of height within the canopy (eq. 1). The authors used this equation to define a metric that could be used in a predictive model of biomass. We believe that it has further potential applications for modelling the behaviour of the LiDAR signal. The following lines provide a description of the Bouvier et al. equation and its interpretation. The gap fraction profile is defined in equation 1 (using the original notations):

$$
P_{k}=\frac{N_{[0 ; z]}}{N_{[0 ; z+d z]}}
$$

where $N_{[0 ; z]}$ refers to the number of returns below $z$, and $N_{[0 ; z+d z]}$ refers to the number of returns below $z+d z$ with $d z$ the thickness of a layer of forest. The equation expresses the number of laser returns that actually reached the layer $z+d z$ and those that passed through the layer $[z ; z+d z] . P_{k}$ represents the gap fraction of the $k^{\text {th }}$ layer.

The gap fraction can be interpreted as the probability, for a single beam carrying contributing energy and reaching the layer $k$, of passing through this layer without interacting with canopy elements. In our model, we consider an alternative event $I$ which expresses the probability $i_{k}$ that a beam reaching layer $k$ interacts with a canopy element in this layer.

$$
i_{k}=1-P_{k}
$$


As described further below, this probability is related to the height distribution of points, which itself can be interpreted as the probability, for a single beam carrying contributing energy, of generating a point in the $k^{\text {th }}$ layer .

Let $p_{k}(\theta)$ be the probability that a beam generates point in the $k^{\text {th }}$ layer at a incidence angle $\theta$. According to equation 2, the probability of interacting with canopy elements in layer $k$ is $i_{k}(\theta)$. If we assume that a beam interacting with a canopy element is always reflected towards the LiDAR sensor with sufficient energy to generate a return (contributing energy), events $I$ and $R$ are equal and $i_{k}=r_{k}$ :

$$
r_{k}(\theta)=1-\frac{\sum_{i=1}^{k-1} p_{i}(\theta)}{\sum_{i=1}^{k} p_{i}(\theta)}
$$

Figure 2 illustrates how equation 3 can be used to calculate the probability of interaction from the height distribution of points in a hypothetical canopy with 5 layers that generate either 20 or 0 returns when sampled vertically. This example shows that to obtain the same number of points in each layer, the canopy must increase in density as the beams approach the ground. Accordingly, the probability of a beam interacting with a canopy element has to increase from top to bottom. The probability associated with the bottom layer is always 1 because of the presence of the ground (gap fraction is 0 ).

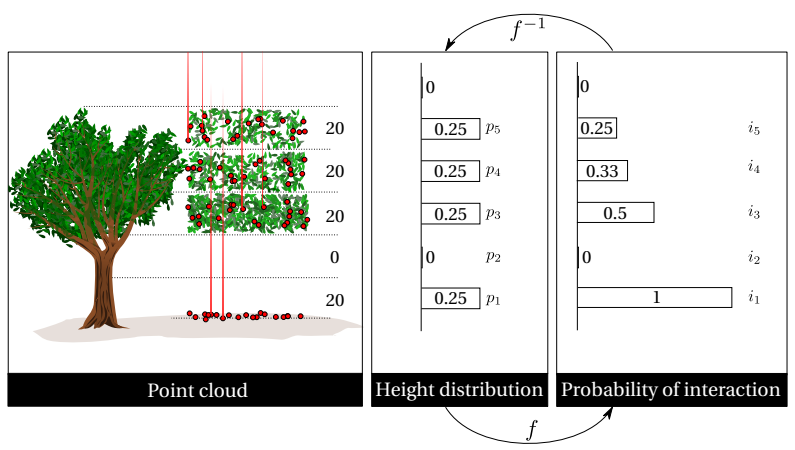

Figure 2: Vertical sampling scenario illustrating how the probability of interaction is calculated from the height distribution of points, and vice versa. Function $f$ (eq 3 ) is used to calculate the probability of interaction from the height distribution, and the inverse function $f^{-1}$ (eq 4 ) is used to recover the height distribution of points from the interaction probabilities.

Figure 2 also illustrates how the interaction probabilities can in turn be used to recover the height distribution 
of points, which is the product of two probabilities - the probability that a beam reaching a layer interacts with it $\left(i_{k}\right)$, and the probability of reaching the layer (i.e. the probability of passing through each previous layer). Thus, the height distribution can be recovered using the reciprocal function, $f^{-1}$ :

$$
p_{k}(\theta)=r_{k}(\theta) \prod_{i=k+1}^{n+1}\left(1-r_{i}(\theta)\right)
$$

with $n$ being the number of layers. Note that for the equation to apply to the case where $k=n$, we added a virtual $n+1$ layer with a probability of interaction of 0 (see AppendixA for explanations and a mathematical demonstration).

\subsection{Effect of incidence angle on the height distribution of points}

When a beam arrives at an angle of $\theta$ degrees, its travel distance through each layer is $1 / \cos (\theta)$ times longer than that of a vertical beam. Thus, the probability of interacting with canopy elements increases with the incidence angle, and there is a corresponding change in the height distribution of points (i.e. the probability that a beam generates a point in any given layer).

As demonstrated further below, an approximate value of the probability of interaction can be calculated from $p_{k}(0)$ by including the factor $1 / \cos (\theta)$ in function $f^{-1}$ (eq. 4$)$. For $k>1, p_{k}(\theta)$ is the probability that a beam arriving at angle $\theta$ generates a point in layer $k$ :

$$
p_{k}(\theta)=\frac{r_{k}(0)}{\cos \theta} \prod_{i=k+1}^{n+1}\left(1-\frac{r_{i}(0)}{\cos \theta}\right)
$$

Thus, the probability of interaction increases in each layer by a factor of $1 / \cos (\theta)$, except the ground layer, which by definition always has a probability of interaction of 1 .

This incidence angle effect is illustrated in figure 3, which shows that increasing the incidence angle from 0 to $45^{\circ}$ increases the probability of interaction by $\sqrt{2}$, thereby shifting the expected height distribution upwards (increasing the number of canopy while decreasing the number of ground points).

To demonstrate that dividing by $\cos (\theta)$ in equation 5 provides a reasonable approximation of the increase in interaction probability at larger incident angles, we must consider the spatial distribution of elements in the canopy. If we assume that the spatial distribution is random, the number of elements in the path of a beam is 


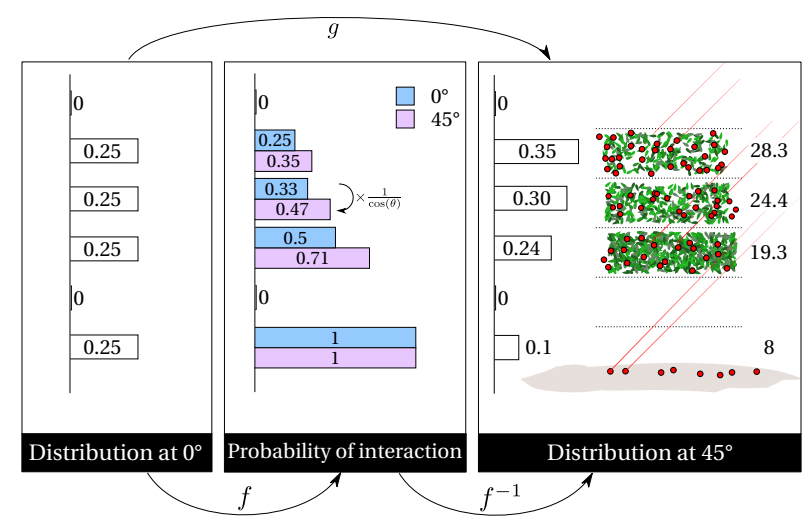

Figure 3: Oblique sampling scenario illustrating how the expected height distribution of points sampled at $45^{\circ}$ is calculated from the height distribution of points observed when sampled vertically (Fig. 1). The function $f$ is used to calculate the interaction probabilities for each layer from the height distribution of points sampled at $0^{\circ}$. The inverse function $f^{-1}$ is used to convert the interaction probabilities back into a height distribution specifying the probabilities that a beam generates a point in any given layer, given that it enters at an angle of $45^{\circ}$. The function $g$ is the composition of $f$ and $f^{-1}\left(g=f^{-1} \circ f\right)$.

a random variable $X$ that follows a Poisson distribution (Nilson, 1971). Thus, the probability, when traveling a distance $d$ through layer $k$, of encountering $n$ elements is:

$$
\mathscr{P}_{k}(X=n)=\frac{d \lambda_{k}^{n} e^{-d \lambda_{k}}}{n !}
$$

and the probability of interacting with at least one canopy element in layer $k$ is $i_{k}$ :

$$
\begin{aligned}
i_{k} & =\mathscr{P}(X>0) \\
& =1-\mathscr{P}(X=0) \\
& =1-e^{-d \lambda_{k}}
\end{aligned}
$$

The quantity $\lambda_{k}$ is related to the density of leaves in the $k^{\text {th }}$ layer, as well as their orientation and spatial distribution. The meaning of this quantity is not required to solve the problem at hand, though readers can refer to Nilson (1971), Campbell and Norman (1990) or the discussion section for more details.

Since $i_{k}=r_{k}$, and the thickness of the layers $\Delta z$ tends towards 0 , we can simplify this expression using the first order Taylor expansion of the exponential near 0 : 


$$
\begin{aligned}
r_{k}(0) & =1-e^{-\Delta z \lambda_{k}} \\
& =1-1+\Delta z \lambda_{k}+O\left(\left(\Delta z \lambda_{k}\right)^{2}\right) \\
& \approx \Delta z \lambda_{k}
\end{aligned}
$$

For oblique angles, the distance $\Delta z$ is increased by the inverse of $\operatorname{cosine} \theta$, and the probability of intersecting at least one element becomes:

$$
r_{k}(\theta)=1-e^{-\frac{\Delta z}{\cos (\theta)} \lambda_{k}}
$$

Finally, Taylor expansion in the neighbourhood of 0 yields the factor included in function $f^{-1}$ (eq 5):

$$
r_{k}(\theta) \approx \frac{\Delta z \lambda_{k}}{\cos (\theta)} \approx \frac{r_{k}(0)}{\cos (\theta)}
$$

Without the first order Taylor expansion the expression would be difficult to manipulate, but it must be noted that the approximation remains valid only if $\frac{\Delta z \lambda_{k}}{\cos (\theta)}$ approaches 0 . In other words, the approximation is correct only for thin layers and narrow incidence angles, as discussed in section 5.

With this approximation, we can then define a function, $g$ (fig 3), using the composition of $f$ and $f^{-1}$ ( $g=$ $f^{-1} \circ f$ ), that enables us to calculate the expected height distribution of points that are sampled obliquely (fig. 3), taking into account the distance required to go through each layer. The source code of this function can be found in the supplementary materials.

\subsection{Observed decrease in number of points per pulse: an unknown effect of incidence angle}

We observed that in addition to increasing the probability of interaction, increasing the incidence angle also decreases the number of returns per beam (figure 4), which could result in an upward shift in the height distribution of points. Thus, we modified our model to provide an empirical way to account for this effect on the height distribution of points. The model does not provide a mechanistic explanation of this phenomenon, though we do provide two plausible explanations in the discussion section.

Given that a point is a spike of energy, the observed decrease in the number of points per pulse represents a decrease either in the amount of energy that is backscattered to the sensor, or in the amplitude of spikes, some 


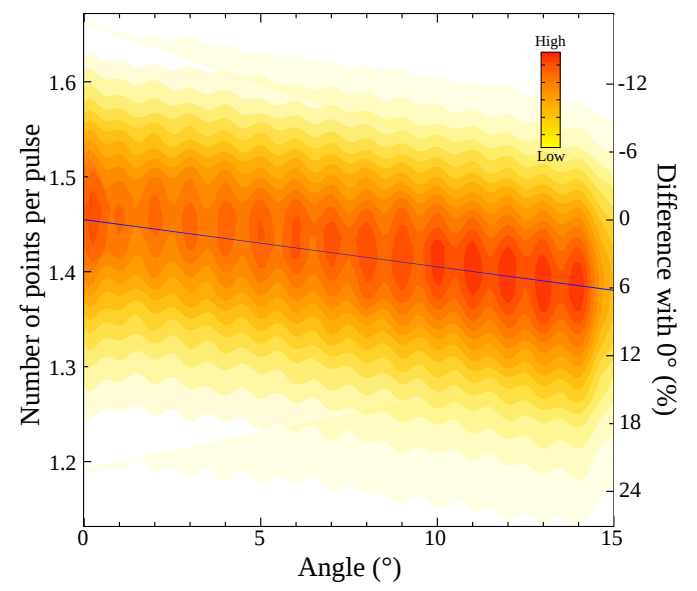

Figure 4: Number of points per pulse as a function of the incidence angle modelled empirically using the linear relationship in 600000 plot rasters covering the all of Haliburton forest. For better readability, the colour scale represents the density of observations. The blue line is the fitted linear regression. The confidence interval is narrower than the line at this scale.

of which become too low to be registered as a point. Thus, the decrease in the number of returns per beam can be represented empirically by allowing foliage to absorb contributing energy, and allowing the probability of absorption to vary with incidence angle, similar to the probability of reflection. This implies relaxing the second assumption presented in section 3.1.

Let $A$ be the event "beam is absorbed" and $a_{k}(\theta)$ its probability in layer $k$ at angle $\theta$. Thus far, we have assumed that a beam interacting with the canopy is always reflected $(I=R)$, but now the energy is either reflected or absorbed: $I=R \cup A$. $R$ and $A$ are two disjoint events, thus:

$$
\begin{aligned}
\mathscr{P}(I) & =\mathscr{P}(R)+\mathscr{P}(A) \\
\Leftrightarrow i_{k}(\theta) & =r_{k}(\theta)+a_{k}(\theta)
\end{aligned}
$$

and equation 5 becomes:

$$
p_{k}(\theta)=\left(\frac{r_{k}(0)}{\cos \theta}+a_{k}(\theta)\right) \prod_{i=k+1}^{n+1}\left(1-\frac{r_{i}(0)}{\cos \theta}-a_{i}(\theta)\right)
$$

Now, the height distribution of points depends on both the incidence angle and vertical variation in absorption. While it is unknown how $a$ changes as a function of $k$, the probability of being absorbed was assumed to be 
proportional to the probability of being reflected. Thus, we introduced a proportionality function, $\alpha$, to describe the variability in absorbed vs reflected energy at different incidence angles:

$$
a_{k}(\theta)=\alpha(\theta) r_{k}(\theta)
$$

While the proportionality function is constant with respect to $k$, the probability of absorption can vary as a linear function of $\theta$, consistent with the pattern shown in figure 4 .

Substituting this term into equation 11 leads to:

$$
i_{k}(\theta)=r_{k}(\theta)(1+\alpha(\theta))
$$

Integrating this new expression into equation 12 leads to the final form of the expression:

$$
p_{k}(\theta)=\left(\frac{r_{k}(0)}{\cos (\theta)}(1+\alpha(\theta))\right) \prod_{i=k+1}^{n+1}\left(1-\frac{r_{i}(0)}{\cos (\theta)}(1+\alpha(\theta))\right)
$$

Using the data shown in figure 4, we can obtain an empirical estimate of the function $\alpha$ from $\epsilon(\theta)$, the overall probability of being absorbed by the canopy at a given incidence angle, as:

$$
\begin{aligned}
& \epsilon(\theta)=\sum_{k=1}^{n} a_{k}(\theta) \\
& \epsilon(\theta)=\alpha(\theta) \sum_{k=1}^{n} r_{k}(\theta) \\
& \alpha(\theta)=\frac{\epsilon(\theta)}{\sum_{k=1}^{n} r_{k}(\theta)}
\end{aligned}
$$

In this equation the only unknown term is the function $\epsilon$. Based on our data and assuming that the relationship is almost linear at least between 0 and $15^{\circ}$, we have:

$$
\epsilon(\theta)=\frac{\theta}{15} \epsilon(15)
$$

with $\epsilon(15) \approx 6 \%=0.06$. Note that $\epsilon$ is the only empirical parameter of our model.

\subsection{Model validation}

The bias of LiDAR metrics can be expected to vary locally because they depend on the local forest structure. However, the number of returns available at the plot scale was insufficient to allow us to accurately apply our 
model to each $400 \mathrm{~m}^{2}$ raster. Thus, to validate our model, we first predicted the average bias of each metric over the whole study area, then compared it to the average bias observed between paired flightlines. For this reason, our model only captures the average bias observed at the scale of the entire forest, not the plot-scale bias that will remain and be manifest as noise.

To implement our model for the whole study area, we first extracted all points sampled at-nadir. Considering the las format specification (ASPRS, 2013) in which the incidence angle information is an integer, nadir $\left(0^{\circ}\right)$ corresponds to a incidence angle ranging from -0.5 to $0.5^{\circ}$. This subset of the data provided an average height distribution of points sampled at-nadir, which was assumed to be representative of the entire forest because the sampling design and the forest structure were completely independent. This reference distribution was then used to recompute the expected height distributions for incidence angles between 0 and $15^{\circ}$, using equations 3 and 5 or 15. Finally, the nine metrics were calculated using each of the distributions, and the expected bias at each angle was calculated as the difference from the reference distribution.

For comparison, we calculated the observed bias by extracting 150000 rasters centred on $(x, y)$ coordinates that were sampled twice in the overlap of adjacent flightlines. The observed bias was then calculated as the difference between the two values obtained for each metric (fig. 5).

\section{Results}

\subsection{Effect of scan angle on the height distribution of points}

As shown in Figure 6, the expected height distribution of points sampled at $30^{\circ}$ includes more returns in the upper canopy than observed at-nadir, which implies more interactions with canopy elements. This pattern is reversed in the lower canopy due to the conservation of energy. The proximity of the green and the blue lines shows that taking into account the reduction of points per beam has a relatively small effect on the expected height distribution of points. In contrast, the difference between the red line and the other two shows that the angular bias described by the probabilistic part of the model is comparatively large.

\subsection{Comparison of observed and expected bias}

Our model accurately reproduced the bias observed in the data (figure 7). Both the sign and magnitude of the bias were correctly predicted for each of the 9 metrics. Including the reduction in the number of points per beam 


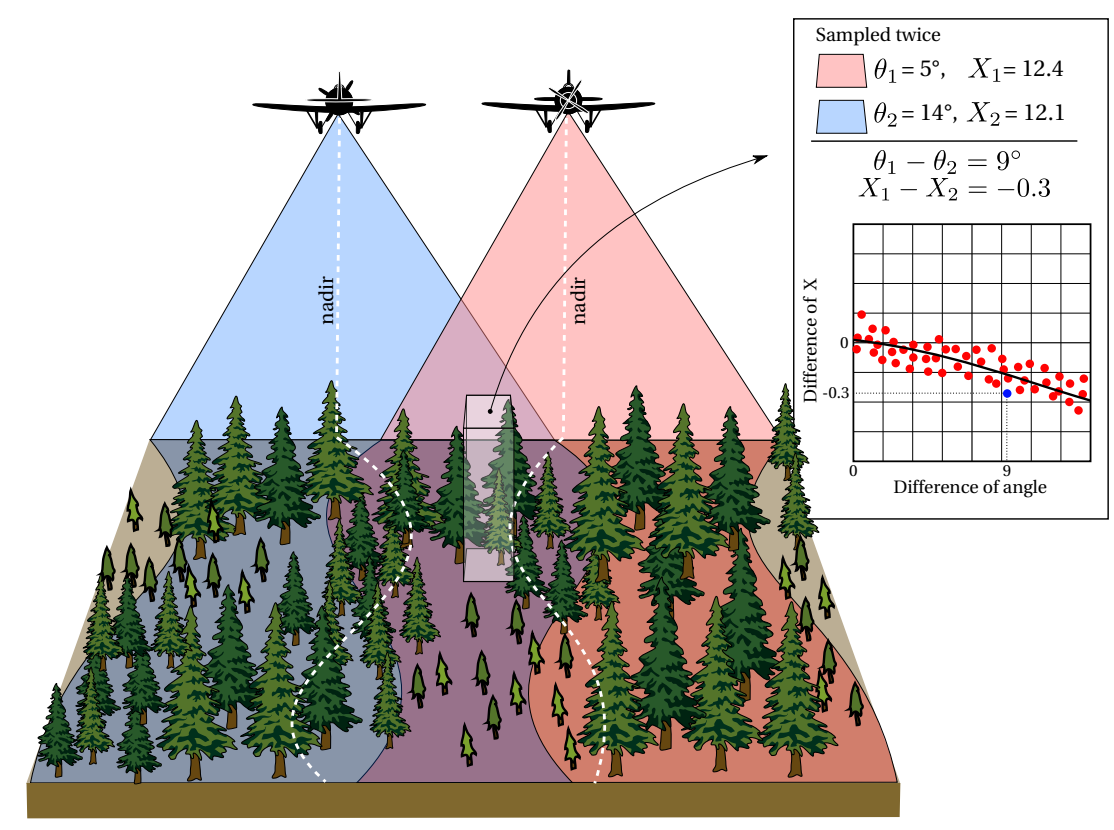

Figure 5: Illustration of the method used to quantify the bias of LiDAR metrics using 150000 plot rasters sampled twice from different flightlines. The bias is the observed difference in the LiDAR metrics obtained from the two flightlines ( $\mathrm{x}$ axis of inset), which varies as function of the difference in incidence angle (y axis of inset). 


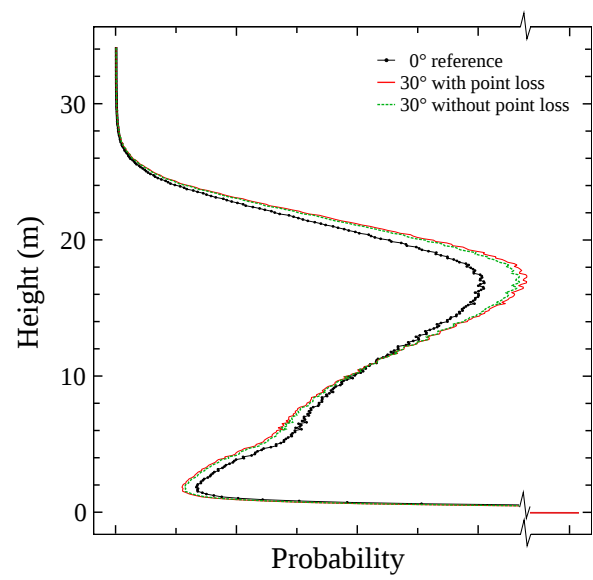

Figure 6: The average height distribution of points sampled at-nadir $\left(0^{\circ}\right)$ and the expected height distribution of points sampled at $30^{\circ}$, with and without considering the fewer number of points per beam (calculated using equations 3 and 15 , respectively). An angle of $30^{\circ}$ was chosen to visualize the magnitude of the predicted angular bias. The histograms appear continuous because they were computed with $1 \mathrm{~cm}$ bins.

only had a small effect on the predicted bias, but doing so brought the expected value closer to the observed value in every case. The observed bias varied considerably from one plot to the next, as shown by the whiskers on either side of the boxes. This is residual bias that is not captured by the model, because it was not implemented at the scale of a plot raster. As mentioned previously, it was implemented using the average height distribution of all the points in the dataset that were sampled at-nadir.

\subsection{The effect of stand structure on bias}

While we were unable to quantify the effect of stand structure empirically, we did calculate the expected bias for three hypothetical stand structures, as shown in Figure 8. The stand structure on the left is unrealistic, but serves to show that the biases can be non monotonic with respect to scan angle. In this example the magnitude of the bias in the standard deviation is low, but increases until $15^{\circ}$ and then decreases until $30^{\circ}$. Thus, despite its apparent simplicity, the model is able to predict complex patterns of bias.

The next two structures are more realistic as they are derived from modified gamma distributions that produce patterns similar to those observed in our data. Both are symmetric and represent typical stand structures in which most returns occur in the lower or upper canopy (but never on the ground). Comparing the patterns of bias in these two stand structures shows that the sign of the bias can switch from positive to negative (or vice versa), depending on the structure of the stand. The relative magnitude of bias can also switch: in the middle panel, for example, 

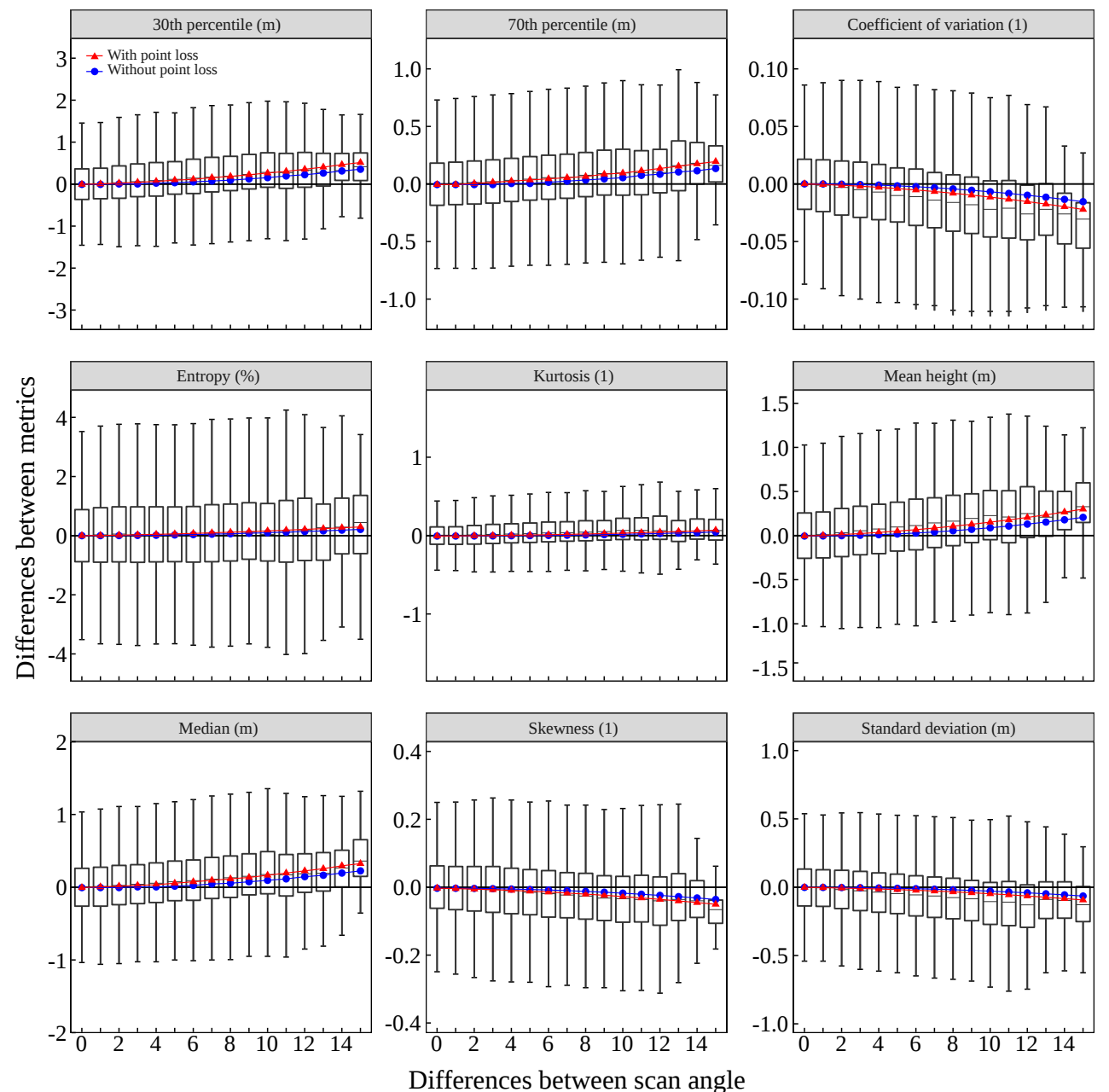

Figure 7: Bias observed in the plot rasters that were sampled twice from different flightlines, as shown in (fig. 5). The observed bias (boxplots) of the nine LiDAR metrics is compared to the the expected bias, both with (red points) and without (blue points) including the reduction in the number of points per beam (calculated using equations 3 and 15, respectively). 

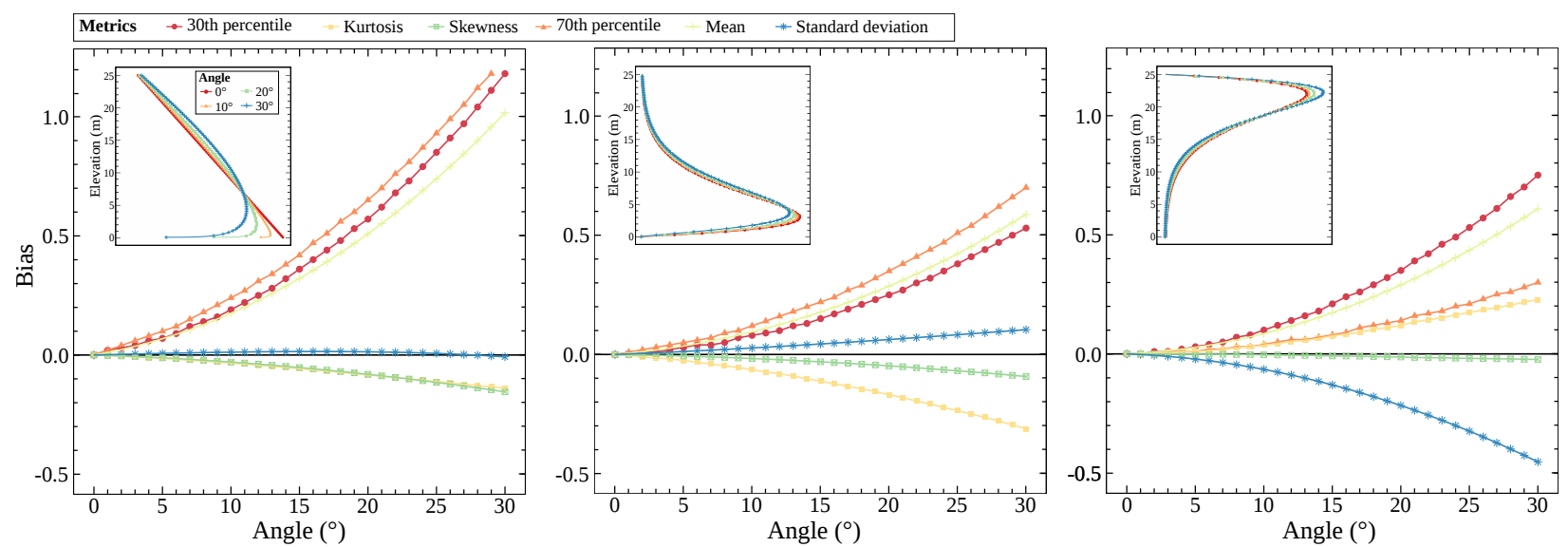

Figure 8: Expected bias in three hypothetical stand structures with a maximum height of $25 \mathrm{~m}$. The expected height distribution of points is plotted in the insets, which show (from left to right) a purely theoretical structure in which the number of returns increases linearly towards the ground, and two more realistic structures in which most returns occur in the lower and upper layers of the canopy. The units of the main $y$ axis are either in meters or dimensionless (depending on the metric units).

the 30th percentile is more biased than the 70th percentile, while the opposite is observed in the right-hand panel. The only constant pattern is the monotonic increase in bias for the height percentiles and the mean height, which was expected at the model development stage.

\section{Discussion}

\subsection{Effect on forest resource inventory}

We started from the hypothesis proposed by Goodwin et al. (2007) and Disney et al. (2010) that the increased travel distance through the canopy increases the probability a beam is reflected as incidence angle increase and we modelled that effect. Our model accurately reproduced the observed bias despite our simplifying assumption that canopy elements are randomly distributed in space. This likely reflects the fact that canopies are relatively continuous in northern hardwood forests. By enabling prediction of the bias attributable to incidence angle for various metrics, our model can be used to normalize LiDAR datasets acquired in forests that meet the assumptions made to build the model.

In our study the average overestimation of the mean height reached $40 \mathrm{~cm}$ at $15^{\circ}$. Bias of this magnitude could conceivably affect the accuracy of area-based models that use LiDAR metrics to predict other stand-level variables of interest, such as stand height, wood volume or aboveground biomass. However, we cannot make general 
statements about the influence of bias on the accuracy of area-based models because it is highly dependent on which LiDAR statistics are used. The bias of some metrics may compensate for one another, which could explain the absence of significant effects in the study of Næsset (1997), while bias may be cumulative in other cases. Our study also showed that the effects of incidence angle depend on the forest structure, which implies that the practical importance of the phenomenon described in our study is very site specific.

An important term in the model is the inverse of the cosine, which renders bias non-linear with respect to incidence angle, indicating that there is a threshold angle beyond which the effect becomes extremely strong. For metrics expressed in meters in our analysis, differences began to reach values larger than one meter at $30^{\circ}$. However, caution must be used in extrapolating to larger angles because of the Taylor expansion that was used to linearize the expression. As the cosine tends towards 0 when $\theta$ tends towards $90^{\circ}$, the term $\frac{\Delta z \lambda_{k}(\theta)}{\cos (\theta)}$ tends towards an undetermined limit. Therefore, the Taylor expansion cannot be used for any combination of variables that make $\frac{\Delta z \lambda_{k}}{\cos (\theta)}$ depart too far from 0 , in which case the equation is no longer amenable to analysis.

In reality, bias would not reach the maximum value associated with the largest incidence angle due to the overlap between flightlines. Approximately $30 \%$ of our area was surveyed in two flightlines, which implies that plots were rarely sampled with a single, large incidence angle. In our dataset, a plot sampled at the maximum angle of $15^{\circ}$ in one flightline was likely to have been scanned at a lower angle of about $10^{\circ}$, for example, in a separate flightline. This should limit the effects of the incidence angle in practice. Having points from two amalgamated flightlines would alter their vertical distribution in a way that is a linear combination of the two effects weighted by the respective local point densities (see supplementary materials).

For future practical applications, we recommend that users first determine whether or not the effect of incidence angle can be neglected. This can be achieved by analysing each flightline separately and comparing the metrics obtained from different angles.

\subsection{Linearity of the observed bias}

We quantified the expected bias at a given angle as the difference between two metrics, one of which was calculated using a $0^{\circ}$ reference distribution - the average height distribution of points sampled at-nadir (Figure 6). In contrast, the two metrics we used to quantify the observed bias were both calculated using rasters that may have been sampled obliquely (fig. 5). This implies that a incidence angle difference of $4^{\circ}$, for example, can 
originate from rasters sampled at 0 and $4^{\circ}$, but also 1 and $5^{\circ}, 8$ and $12^{\circ}, 10$ and $14^{\circ}$ etc. The same applies to all incidence angle differences except $15^{\circ}$, which was necessarily the result of a raster sampled at-nadir and another one sampled at $15^{\circ}$ because the maximum incidence angle was $15^{\circ}$.

Because our model always used $0^{\circ}$ as a reference, our comparison of the expected and observed bias (figure 7) is only valid if the effects are linear. Figure 7 showed this was not strictly true, but within the range of our observations the angles are small enough, and the effects linear enough, that we consider the comparison to be valid. Using all the rasters sampled in two flightlines was necessary to obtain enough data to highlight the overall pattern hidden in the noise. If we had only used pairs that included one raster sampled at-nadir, the dataset of observed variation would have decreased from 150000 to only 600 rasters, which was insufficient to show the signal.

\subsection{Accounting for the reduction of number of returns per beam}

Despite the limited magnitude of this effect on the expected bias, the data clearly showed that the number of points per pulse decreases with increasing incidence angle. To our knowledge, this phenomenon has not yet been reported in the scientific literature. One hypothesis is that as incidence angle increases, a pulse is more likely to be intercepted by vertical tree trunks because they become more exposed. Trunks are covered in bark that is more likely than foliage to absorb energy at $1064 \mathrm{~nm}$. Thus, an oblique beam is less likely to be reflected when it encounters a tree trunk, and if absorbed there will be no subsequent returns. According to this hypothesis, the average number of points per beam can only decrease as a function of the incidence angle. Moreover the average intensity of first returns should be constant with respect to the incidence angle (if a range correction is applied).

Another hypothesis is that the probability of being absorbed by foliage increases with incidence angle. This can happen if the spatial distribution and orientation of leaves are not random. However, in contrast to the previous hypothesis, this could also lead to an increase in the number of pulses per beam in certain circumstances.

To appreciate why, consider the parameter $\lambda_{k}$ that was first introduced in equation 6 . $\lambda_{k}$ is the product of the leaf area density $\mu$, the $G$ function and the clumping factor $\Omega$ (e.g. Nilson, 1971; Campbell and Norman, 1990). Thus, $\lambda_{k}$ is not only a function of elevation $(k)$ but also a function of incidence angle $\theta$ :

$$
\lambda_{k}(\theta)=\mu_{k} G_{k}(\theta) \Omega_{k}(\theta)
$$


However, we assumed that the spatial distribution of foliage is random, in which case $\Omega(\theta)$ equals unity and (given a spherical leaf angle distribution) $G(\theta)=0.5$, for any incidence angle. $\lambda$ was therefore only a function of the elevation $\left(\lambda_{k}\right)$ in equation 6.

If these assumptions are relaxed equation 9 becomes:

$$
\begin{aligned}
i_{k}(\theta) & =\frac{\Delta z \lambda_{k}(\theta)}{\cos (\theta)} \\
& =\frac{\Delta z \mu_{k} G(\theta) \Omega(\theta)}{\cos (\theta)} \\
& =\frac{\Delta z \mu_{k} G(0) \Omega(0) G(\theta) \Omega(\theta)}{\cos (\theta) G(0) \Omega(0)} \\
& =\frac{r_{k}(0)}{\cos (\theta)} \frac{G(\theta) \Omega(\theta)}{G(0) \Omega(0)}
\end{aligned}
$$

Comparing the above to equation 14 ,

$$
i_{k}(\theta)=\frac{r_{k}(0)}{\cos (\theta)}(1+\alpha(\theta))
$$

we note that what is known as the extinction coefficient $K$-the $G$ function multiplied by the clumping factoris the function $1+\alpha$ in our model. The $G$ function depicts the azimuthal angle distribution of the foliage. $G$ can be an increasing or decreasing function of the view angle and $K$ as well. Thus, contrary to the first hypothesis, this second hypothesis allows the bias to be positive or negative i.e. either an increasing or decreasing number of returns per beam.

Because both hypotheses lead to the same mathematical formulation i.e. a factor that multiplies the term $\frac{r_{k}}{\cos (\theta)}$, further analysis of the reduction in the number of points per beam in other datasets is required to distinguish between them. If decreases in number of returns per pulse are consistently observed at oblique incident angles, then it could be likely attributed to absorption by bark.

In our model, absorption was proportional to the density of canopy elements and the proportionality function (eq. 13) was constant with respect to height. This was sufficient to reproduce the behaviour of the data, but in reality it is likely that this coefficient varies between layers. A deeper inspection of the sequence of multiple returns would be necessary to refine our understanding of the reduction in the number of points per beam. In addition to evaluating how this reduction is distributed along the sequence of returns, it would be interesting to 
examine how it varies with height. For example, Næsset (2009) found that that single echoes tend to occur in the densest parts of the tree crowns. Since our model assumes the forest is perceived to be denser off-nadir, the results are therefore compatible.

Over and above all the considerations presented in this section, it must be highlighted that the part of the model accounting for the reduction in the number of returns per beam remains only an empirical add-on. It should not be considered as an intrinsic part of the model that was developed using a hypothesis-driven approach. Instead, we have used this model to propose one way to address the question of point loss. Other explanations can be proposed to explain this phenomenon, such as a loss of energy backscattered due to the increasing path length, for example.

\subsection{Model applicability}

An important limit of our study is that the model was only validated using a single dataset from a northern hardwood forest, and thus for a specific instrument and specific survey settings. Despite this, we believe the model has more general applicability over any type of forest that meets our initial assumptions.

Under our hypothesis-driven approach, our model was derived from a few initial assumptions made about the forest canopy structure and the way energy is spread and backscattered, independently of any site- or device-specific principles. The model is self-contained and does not rely on empirical data. This is the key to justify the empirical validation using a single dataset, which is only deemed to provide a demonstration that the model can be applicable in reality, in one forest type that meets our initial assumptions. The fact the model fits well with our validation dataset provides a good indication that it can offer a plausible representation of the physical reality.

We therefore expect a similar applicability in other forest types that meet the same assumptions. However, with the infinity of forest structures that can be found globally, it would not be possible to provide an exhaustive analysis of the limits of applicability of the model. For this reason, our approach was to provide 1) one example and 2) a source code that enables future users to determine if the model applies or not to their specific context.

Listing all types of forest canopies that could be adequately represented as a set of horizontal turbid layers is 
beyond the scope of this study. However, we believe the model may be applicable to any closed-canopy forests dominated by broadleaved trees. This includes temperate hardwood forests, but also to tropical humid or even dry tropical forests. Obviously, model applicability remains to be empirically demonstrated in other ecosystems.

\subsection{Alternative approaches for discontinuous canopies}

Our initial assumption that the forest canopy can be represented as a set of horizontal turbid layers would not be valid for forests with a clumped canopy structure such as conifer or savannah canopies. Coniferous forests, for example, exhibit hierarchical clumping structure at different levels (Wenge et al., 1997), and individual conifers are more analogous to large, solid geometrical objects (Li and Strahler, 1985). This suggests that the influence of incidence angle is determined more by geometrical effects than by probabilistic effects.

Our model is analogous to radiative transfer (RT) models because it describes the probability of interacting with components of turbid homogeneous horizontal layers. Another way to model canopies is the geometrical optic (GO) approach, which was first developed for discontinuous conifer canopies that can be represented as an assemblage of three-dimensional, solid objects. The conceptual foundations of both the RT and GO approach were formalized decades ago (e.g. Li and Strahler, 1985; Strahler and Jupp, 1990). To develop a GO model of incidence angle effects discontinuous canopies, the first step would be to transfer existing GO equations to LiDAR applications, similarly to what we attempted in this study with the RT approach. A more advanced approach could even rely on equations that use both GO and RT principles (GORT approach) in the spirit of studies proposed by Wenge et al. (1997) or Haverd et al. (2012).

\section{Conclusion}

We examined the changes in the height distribution of returns that result from increasing the incidence angle, and hence both the distance a beam travels through the canopy and the probability the beam is reflected by the canopy. We developed a conceptual framework for understanding and predicting the resulting bias of LiDAR metrics, and demonstrated that our model accurately reproduced the bias calculated for northern hardwoods with relatively continuous canopies. The model allows a point distribution sampled at-nadir to be recomputed "as if it were sampled at another incidence angle".

The model also suggests that the non-random spatial distribution of foliage may be responsible for fewer returns per beam at large incidence angles. Alternatively, this may reflect the fact that oblique pulses are more 
likely to be intercepted by vertical tree trunks, resulting in the end of a return sequence. Nevertheless, our model predicts the number of points per beam has a small effect on the height distribution of points, compared to increasing the length of the path a beam travels through the canopy.

\section{Acknowledgement}

This research was funded by AWARE (the Assessment of Wood Attributes from Remote Sensing NSERC CRDPJ462973-14; grantee Prof Nicholas Coops, UBC), in collaboration with Canadian Wood Fiber Centre (CWFC), FP-Innovations and Tembec.

\section{References}

Anderson, E. S., Thompson, J. A., Crouse, D. A., and Austin, R. E. (2006). Horizontal resolution and data density effects on remotely sensed LIDAR-based DEM. Geoderma, 132(3-4):406-415.

ASPRS (2013). LAS SPECIFICATION VERSION 1.4 - R13. The American Society for Photogrammetry and Remote Sensing.

Bouvier, M., Durrieu, S., Fournier, R. a., and Renaud, J.-p. (2015). Remote Sensing of Environment Generalizing predictive models of forest inventory attributes using an area-based approach with airborne LiDAR data. Remote Sensing of Environment, 156:322-334.

Campbell, G. and Norman, J. (1990). The description and measurement of plant canopy structure, volume 31. Cambridge University Press.

Disney, M., Kalogirou, V., Lewis, P., Prieto-Blanco, a., Hancock, S., and Pfeifer, M. (2010). Simulating the impact of discrete-return lidar system and survey characteristics over young conifer and broadleaf forests. Remote Sensing of Environment, 114(7):1546-1560.

Evans, J. S., Hudak, A. T., Faux, R., and Smith, A. M. S. (2009). Discrete return lidar in natural resources: Recommendations for project planning, data processing, and deliverables. Remote Sensing, 1(4):776-794.

Gleason, C. J. and Im, J. (2012). Forest biomass estimation from airborne LiDAR data using machine learning approaches. Remote Sensing of Environment, 125:80-91. 
Gobakken, T. and Næsset, E. (2008). Assessing effects of laser point density, ground sampling intensity, and field sample plot size on biophysical stand properties derived from airborne laser scanner data. Canadian Journal of Forest Research, 38(5):1095-1109.

Goodwin, N., Coops, N., and Culvenor, D. (2007). Development of a simulation model to predict LiDAR interception in forested environments. Remote Sensing of Environment, 111(4):481-492.

Haverd, V., Lovell, J., Cuntz, M., Jupp, D., Newnham, G., and Sea, W. (2012). The canopy semi-analytic pgap and radiative transfer (canspart) model: Formulation and application. Agricultural and Forest Meteorology, 160:14 35.

Holmgren, J. (2004). Prediction of tree height, basal area and stem volume in forest stands using airborne laser scanning. Scandinavian Journal of Forest Research, 19(6):543-553.

Holmgren, J., Nilsson, M., and Olsson, H. (2003a). Estimation of tree height and stem volume on plots using airborne laser scanning. Forest Science, 49(3):419-428.

Holmgren, J., Nilsson, M., and Olsson, H. (2003b). Simulating the effects of lidar scanning angle for estimation of mean tree height and canopy closure. Canadian Journal of Remote Sensing, 29(5):623-632.

Ioki, K., Imanishi, J., Sasaki, T., Morimoto, Y., and Kitada, K. (2009). Estimating stand volume in broad-leaved forest using discrete-return LiDAR: plot-based approach. Landscape and Ecological Engineering, 6(1):29-36.

Jakubowski, M. K., Guo, Q., and Kelly, M. (2013). Tradeoffs between lidar pulse density and forest measurement accuracy. Remote Sensing of Environment, 130:245-253.

Kwak, D.-A., Lee, W.-K., Cho, H.-K., Lee, S.-H., Son, Y., Kafatos, M., and Kim, S.-R. (2010). Estimating stem volume and biomass of Pinus koraiensis using LiDAR data. Journal of plant research, 123(4):421-32.

Li, X. and Strahler, A. (1985). Geometric-optimal modeling of a conifer forest canopy. IEEE Transactions on Geoscience and Remote Sensing, 23(5):705-721.

Lim, K., Hopkinson, C., and Treitz, P. (2008). Examining the effects of sampling point densities. Forestry Chronicle, 84(6):876-885.

Lim, K., Treitz, P., Baldwin, K., Morrison, I., and Green, J. (2014). Lidar remote sensing of biophysical properties of tolerant northern hardwood forests. Canadian Journal of Remote Sensing, 29(5):658-678. 
Lovell, J., Jupp, D., Newnham, G., Coops, N., and Culvenor, D. (2005). Simulation study for finding optimal lidar acquisition parameters for forest height retrieval. Forest Ecology and Management, 214(1-3):398-412.

Maltamo, M., Næsset, E., and Vauhkonen, J. (2014). Forestry Applications of Airborne Laser Scanning: Concepts and Case Studies. Springer.

Montaghi, A. (2013). Effect of scanning angle on vegetation metrics derived from a nationwide Airborne Laser Scanning acquisition. Canadian Journal of Remote Sensing, 39(sup1):S152-S173.

Morsdorf, F., Frey, O., Meier, E., Itten, K. I., and Allgöwer, B. (2008). Assessment of the influence of flying altitude and scan angle on biophysical vegetation products derived from airborne laser scanning. International Journal of Remote Sensing, 29(5):1387-1406.

Morsdorf, F., Meier, E., Kötz, B., Itten, K. I., Dobbertin, M., and Allgöwer, B. (2004). LIDAR-based geometric reconstruction of boreal type forest stands at single tree level for forest and wildland fire management. Remote Sensing of Environment, 92(3):353-362.

Næsset, E. (1997). Determination of mean tree height of forest stands using airborne laser scanner data. \{ISPRS\} Journal of Photogrammetry and Remote Sensing, 52(2):49-56.

Næsset, E. (2009). Effects of different sensors, flying altitudes, and pulse repetition frequencies on forest canopy metrics and biophysical stand properties derived from small-footprint airborne laser data. Remote Sensing of Environment, 113(1):148-159.

Nilson, T. (1971). A theoretical analysis of the frequency of gaps in plant stands. Agricultural meteorology, 8:25-38. Pirotti, F. and Tarolli, P. (2010). Suitability of LiDAR point density and derived landform curvature maps for channel network extraction. Hydrological Processes, 24(9):1187-1197.

Popescu, S. C., Wynne, R. H., and Nelson, R. F. (2002). Estimating plot-level tree heights with lidar: local filtering with a canopy-height based variable window size. Computers and Electronics in Agriculture, 37(1-3):71-95.

Pyysalo, U. and Hyyppä, H. (2002). Reconstructing tree crowns from laser scanner data for feature extraction. International Archives Of Photogrammetry Remote Sensing And Spatial Information Sciences, 34:218-221.

R Core Team (2015). R: A Language and Environment for Statistical Computing. R Foundation for Statistical Computing, Vienna, Austria. 
Reitberger, J., Schnörr, C., Krzystek, P., and Stilla, U. (2009). 3D segmentation of single trees exploiting full waveform LIDAR data. ISPRS Journal of Photogrammetry and Remote Sensing, 64(6):561-574.

Roussel, J.-R. and Auty, D. (2017). lidR: Airborne LiDAR Data Manipulation and Visualization for Forestry Applications. R package version 1.1.0, avaible at https ://github.com/Jean-Romain/lidR.

Roussel, J.-R., Béland, M., Caspersen, J., and Achim, A. (2017). Probabilistic model for canopy correction taking into account pulse density, footprint size, overlaps and plane pitch: the local maximum algorithm and its side effects.

Strahler, A. H. and Jupp, D. L. B. (1990). Modeling bidirectional reflectance of forests and woodlands using boolean models and geometric optics. Remote Sensing of Environment, 34(3):153-166.

Thomas, V., Treitz, P., McCaughey, J. H., and Morrison, I. (2006). Mapping stand-level forest biophysical variables for a mixedwood boreal forest using lidar: an examination of scanning density. Canadian Journal of Forest Research, 36(1):34-47.

van Ewijk, K. Y., Treitz, P. M., and Scott, N. A. (2011). Characterizing Forest Succession in Central Ontario using Lidar-derived Indices. Photogrammetric Engineering and Remote Sensing, 77(3):261-269.

Vauhkonen, J., Maltamo, M., McRoberts, R. E., and Næsset, E. (2014). Forestry Applications of Airborne Laser Scanning: Concepts and Case Studies, chapter Introduction to Forestry Applications of Airborne Laser Scanning, pages 1-16. Springer Netherlands, Dordrecht.

Vega, C., Hamrouni, a., El Mokhtari, S., Morel, J., Bock, J., Renaud, J.-P., Bouvier, M., and Durrieu, S. (2014). PTrees: A point-based approach to forest tree extraction from lidar data. International Journal of Applied Earth Observation and Geoinformation, 33:98-108.

Wenge, N., Xiaowen, L., Woodcock, C. E., Roujean, J.-L., and Davis, R. E. (1997). Transmission of solar radiation in boreal conifer forests: Measurements and models. Journal of Geophysical Research, 102:29555—-29566.

White, J. C., Wulder, M. A., Varhola, A., Vastaranta, M., Coops, N. C., Cook, B. D., Pitt, D., Woods, M., et al. (2013). A best practices guide for generating forest inventory attributes from airborne laser scanning data using an area-based approach. For. Chron, 89(722723):5.

Woods, M., Pitt, D., Penner, M., Lim, K., Nesbitt, D., Etheridge, D., and Treitz, P. (2011). Operational implementation of a lidar inventory in boreal ontario. The Forestry Chronicle, 87(4):512-528. 
${ }_{622}$ Yao, W., Krzystek, P., and Heurich, M. (2012). Tree species classification and estimation of stem volume and 623 DBH based on single tree extraction by exploiting airborne full-waveform LiDAR data. Remote Sensing of 624 Environment, 123:368-380. 
632 Then,

(Then, sense:

\section{AppendixA. Function $f^{-1}$, basic form}

We try to demonstrate that the function $f^{-1}$ can be written:

$$
\forall k \in \llbracket 1, n \rrbracket, p_{k}=r_{k} \prod_{i=k+1}^{n+1}\left(1-r_{i}\right)
$$

Let's consider $n$ layers, with the ground layer being layer 1, and the highest layer the layer $n$. The probability to find a beam travelling thought the layer $k$ is $q_{k}$. This beam travelling through layer $k$ interacts with canopy components within that layer with a probability $r_{k}$. The probability to generate a point in the layer $k$ is:

$$
p_{k}=r_{k} q_{k}
$$

The probability $q_{k}$ is the probability that the beam passed through each previous layer without interacting with canopy components.

$$
\begin{aligned}
q_{k} & =\mathscr{P}\left(\bar{R}_{k+1} \cap \bar{R}_{k+2} \cap \ldots \cap \bar{R}_{n}\right) \\
& =\left(1-r_{k+1}\right) \times\left(1-r_{k+2}\right) \times \ldots \times\left(1-r_{n}\right) \\
& =\prod_{i=k+1}^{n}\left(1-r_{i}\right)
\end{aligned}
$$

$$
p_{k}=r_{k} \prod_{i=k+1}^{n}\left(1-r_{i}\right)
$$

The case where $k=n$ is a particular case which does not follow the rule because the formula does not make

$$
p_{n}=r_{n} \prod_{i=n+1}^{n}\left(1-r_{i}\right)
$$

Adding a virtual layer $n+1$ with a probability $r_{n+1}$ of interaction of 0 solves the issue adding a neutral element into the product:

$$
p_{k}=r_{k} \prod_{i=k+1}^{n+1}\left(1-r_{i}\right)
$$

